\title{
Publisher Correction: The adenosine pathway in immuno-oncology
}

Bertrand Allard, David Allard, Laurence Buisseret (1) and John Stagg (1)

Nature Reviews Clinical Oncology (2020) https://doi.org/10.1038/s41571-020-0382-2 Published online 08 June 2020

The originally published article contained an error in Table 1, in which oleclumab was incorrectly described as an anti-CD79 monoclonal antibody $(\mathrm{mAb})$ instead of an anti-CD73 mAb. This editorial error has been corrected in the HTML and PDF versions of the manuscript to reflect that oleclumab in an anti-CD73 mAb.

https://doi.org/10.1038/s41571-020-0415-x I Published online 17 July 2020

(c) Springer Nature Limited 2020 